\title{
ON COMMUTANT OF DIFFERENTIATION AND TRANSLATION OPERATORS IN WEIGHTED SPACES OF ENTIRE FUNCTIONS
}

\author{
O.A. IVANOVA, S.N. MELIKHOV, YU.N. MELIKHOV
}

\begin{abstract}
We describe continuous linear operators acting in a countable inductive limit $E$ of weighted Fréchet spaces of entire functions of several complex variables and commuting in these spaces with systems of partial differentiation and translation operators. Under the made assumptions, the commutants of the systems of differentiation and translation operators coincide. They consist of convolution operators defined by an arbitrary continuous linear functional on $E$. At that, we do not assume that the set of the polynomials is dense in $E$. In the space $E^{\prime}$ topological dual to $E$, we introduce the natural multiplication. Under this multiplication, the algebra $E^{\prime}$ is isomorphic to the aforementioned commutant with the usual multiplication, which is the composition of the operators. This isomorphism is also topological if $E^{\prime}$ is equipped by the weak topology, while the commutant is equipped by the weak operator topology. This implies that the set of the polynomials of the differentiation operators is dense in the commutant with topology of pointwise convergence. We also study the possibility of representing an operator in the commutant as an infinite order differential operator with constant coefficients. We prove the immediate continuity of linear operators commuting with all differentiation operators in a weighted (LF)-space of entire functions isomorphic via Fourier-Laplace transform to the space of infinitely differentiable functions compactly supported in a real multi-dimensional space.
\end{abstract}

Keywords: differentiation operator, translation operator, commutant, weighted space of entire functions.

Mathematics Subject Classification: 30D15, 47B38, 46E10

\section{INTRODUCTION}

A lot of works were devoted to studying operators commuting with the differentiation in the spaces of analytic functions. Continuous linear operators commuting with the differentiation and translation operators in the spaces of functions analytic in the domains in $\mathbb{C}$ and $\mathbb{C}^{N}$, were studied in [1], 3], 10], [15] (see the references in [5, Sect. 2.8]). The commutants of differentation operators in weighted spaces $E$ of entire functions were studied in [6], [16]. In the space of entire in $\mathbb{C}^{N}$ functions of exponential type being dual to the space of functions holomorphic in a domain in $\mathbb{C}^{N}$ such description was obtained in [11], see also [12, Sect. 2, Prop. 1]. If the corresponding domain is convex, then this space with the natural topology is a weighted (LB)-space. In [7, Ch. 9, Sect. 7] there were studied linear and continuous in some sense operators commuting with the differentiation in a special space $P^{*}$ of entire in $\mathbb{C}$ functions. In all considered situations the operators commuting with the differentiation operators turn out to be the convolution operators, in particular, differential operators of infinite order playing an important role in applications.

O.A. Ivanova, S.N. Melikhov, Yu.N. Melikhov, On COmmutant of Differentiation and transLATION OPERATORS IN WEIGHTED SPACES OF ENTIRE FUNCTIONS.

(C) Ivanova O.A., Melikhov S.N., Melikhov Yu.N. 2017.

Submitted June 30, $201 \%$. 
In the present work we describe the commutants $\mathcal{K}(\partial)$ and $\mathcal{K}(\tau)$ of the systems of partial differentiation operators and translation operators, respectively, in the countable inductive limit $E$ of weighted Fréchet spaces of entire in $\mathbb{C}^{N}$ functions. Such spaces $E$ are intensively used. They arise while realizing various spaces by Fourier-Laplace transform and its analogues. In all previous studies the density of polynomials in the corresponding spaces was employed essentially in description of $\mathcal{K}(\partial)$. Here this property is not supposed to hold and we do not assume that $E$ contains polynomials. This is possible thanks to the density of the system of the functionals $\varphi_{\alpha}: f \mapsto \partial^{\alpha} f(0), \alpha \in \mathbb{N}_{0}^{N}$ in the space $E^{\prime}$ topological dual to $E$ with the topology consistent with the duality between $E^{\prime}$ and $E$, see Section 1. In another situation this approach was employed in [2] while studying the operators commuting with the Pommiez operator. As in many other cases, under the made assumptions, $\mathcal{K}(\partial)$ coincides with the set $\mathcal{K}(\tau)$. Among these assumptions, the most restrictive one is Condition (V2) close to the semi-additivity of the weights defining the space $E$. The description of the operators in $\mathcal{K}(\tau)$ is simple thanks to the possibility of swapping the argument and translation vector in the commutation identity. The operators in $\mathcal{K}(\partial)=\mathcal{K}(\tau)$ are only those acting by the rule $f \mapsto \varphi_{t}(f(t+z)), z \in \mathbb{C}^{N}, f \in E$, where $\varphi$ is an arbitrary continuous linear functional on $E$, that is, the convolution operators. As above, the arbitrariness of the functional $\varphi \in E^{\prime}$ ensures Condition (V2). Condition (V2) is in some sense essential for this as it is shown by the example of the space $E$ of the Fourier-Laplace transforms $\mathcal{F}$ of all functions in $\mathcal{D}$, which is the space of all infinitely differentiable compactly supported in $\mathbb{R}^{N}$ functions. In this case the sets $\mathcal{K}(\partial)$ and $\mathcal{K}(\tau)$ also coincide, their elements are the convolution operators but $\varphi$ can range only in the eigenspace $\left(\mathcal{F}^{\prime}\right)^{-1}\left(C^{\infty}\left(\mathbb{R}^{N}\right)\right)$ of the space $E^{\prime}$. Here $\mathcal{F}^{\prime}: E^{\prime} \rightarrow \mathcal{D}^{\prime}$ stands for the operator adjoint for $\mathcal{F}: \mathcal{D} \rightarrow E$; in this case condition (V1) holds, while (V2) fails. At that, one proves the result on immediate continuity of the linear operators in $\mathcal{D}$ commuting with all operators of multiplication by independent variables. Due to the properties of the Fourier-Laplace transform, this implies the immediate continuity of linear operators $E \simeq \mathcal{D}$ commuting with all partial differentiations.

In the topological dual space $E^{\prime}$ for $E$ (if the weights satisfy Condition (V2)) we naturally introduce the multiplication $\odot$. The algebra $\left(E^{\prime}, \odot\right)$ is isomorphic to the algebra $\mathcal{K}(\partial)$ with the usual multiplication, which is the composition of the operators. The corresponding isomorphism is also topological if $E^{\prime}$ is equipped with the weak topology and $\mathcal{K}(\partial)$ is equipped with the weak operator topology. Such weak topological isomorphy of $E^{\prime}$ and $\mathcal{K}(\partial)$ allows us to show that the set of the polynomials of the partial differentiation operators is dense in the space $\mathcal{K}(\partial)$ equipped with the topology of pointwise convergence and to study the possibility of representing the operators in $\mathcal{K}(\partial)$ as infinite order differential operators with constants coefficients.

\section{DESCRIPTION OF THE COMMUTANT OF THE SYSTEM OF PARTIAL DIFFERENTIATION OPERATORS AND TRANSLATIONALLY INVARIANT OPERATORS}

1.1. Main result. Let $N \in \mathbb{N} ; v_{n, k}: \mathbb{C}^{N} \rightarrow \mathbb{R}, n, k \in \mathbb{N}$, be a double sequence of continuous functions such that

$$
v_{n, k+1} \leqslant v_{n, k} \leqslant v_{n+1, k}, \quad n, k \in \mathbb{N},
$$

in $\mathbb{C}^{N}$. For $n, k \in \mathbb{N}$ we introduce weighted Banach spaces of entire functions

$$
E_{n, k}:=\left\{f \in H\left(\mathbb{C}^{N}\right) \mid\|f\|_{n, k}:=\sup _{z \in \mathbb{C}^{N}} \frac{|f(z)|}{\exp \left(v_{n, k}(z)\right)}<+\infty\right\}
$$

and Fréchet spaces $E_{n}:=\bigcap_{k \in \mathbb{N}} E_{n, k}$. The topology in $E_{n}$ is induced by the sequence of the norms $\|\cdot\|_{n, k}, k \in \mathbb{N}$. At that, the symbol $H\left(\mathbb{C}^{N}\right)$ stands for the space of all entire in $\mathbb{C}^{N}$ functions.

Each space $E_{n}$ is continuously embedded into $E_{n+1}$.

We let $E:=\bigcup_{n \in \mathbb{N}} E_{n}$. In $E$ we introduce the topology of the inductive limit of Fréchet spaces $E_{n}, n \in \mathbb{N}$, with respect to the embedding of $E_{n}$ into $E$. 
Let $|z|:=\left(\sum_{j=1}^{N}\left|z_{j}\right|^{2}\right)^{1 / 2}$ for $z=\left(z_{j}\right)_{j=1}^{N} \in \mathbb{C}^{N}$. We introduce the conditions:

(V1) For each $n$ there exists $m$ such that for each $k$ there exists $s$ such that

$$
\lim _{|z| \rightarrow \infty}\left(\inf _{|t-z| \leqslant 1} v_{m, k}(t)-\sup _{|t-z| \leqslant 1} v_{n, s}(t)\right)=+\infty
$$

and

(V2) For each $n$ there exists $m$ such that for each $k$ there exist $s$ and $C<+\infty$ such that for each $t, z \in \mathbb{C}^{N}$ we have

$$
v_{n, s}(t+z) \leqslant v_{m, k}(t)+v_{m, k}(z)+C .
$$

Remark 1. In order to track the dependence of the chosen indices, we shall write Condition (V1) also as "for each $n$ there exists $m(n)$ such that for each $k$ there exists $s(n, k)$ ".

Let $\partial_{j} f:=\frac{\partial f}{\partial z_{j}}, 1 \leqslant j \leqslant N$. We introduce the translation operator

$$
\tau_{z}(f)(t):=f(t+z), z, t \in \mathbb{C}^{N}, f \in E .
$$

Remark 2. Assume that Condition (V1) holds. Then E possesses the following properties: (i) Each operator $\partial_{j}$ maps linearly and continuously $E$ into E. At that, for each $n$ there exists $m$ such that for each $k$ there exist $s$ and $B<+\infty$ obeying

$$
\left\|\partial_{j} f\right\|_{m, k} \leqslant B\|f\|_{n, s}, f \in E_{n}, 1 \leqslant j \leqslant N .
$$

Here $m$ and $s$ can be chosen that same as in Condition (V1).

(ii) For each $z \in \mathbb{C}^{N}$, the operator $\tau_{z}$ maps linearly and continuously $E$ into $E$.

(iii) For each $n \in \mathbb{N}$ there exists $m \in \mathbb{N}$ such that each bounded set in $E_{n}$ is relatively compact in $E_{m}$. And for each $n$, the constant $m$ can be chosen as in Condition (V1).

Proof. Inequality (1) follows the one-dimensional integral Cauchy formula. It implies that for each $n$ each operator $\partial_{j}$ maps continuously $E_{n}$ into $E_{m}$, and hence, $E$ into $E$.

Statement (iii) is implied by Montel's theorem.

The role of Condition (V2) is clarified by Lemma 3.

Let $\mathcal{L}(E)$ be the space of all continuous linear operators in $E$. This is a ring with usual summation and multiplication (composition) of operators. We introduce the commutant $\mathcal{K}(\tau)$ of the system of translation operators in $\mathcal{L}(E)$ :

$$
\mathcal{K}(\tau):=\left\{A \in \mathcal{L}(E) \mid \forall z \in \mathbb{C}^{N} A \tau_{z}=\tau_{z} A \text { in } E\right\}
$$

and the commutant $\mathcal{K}(\partial)$ of the system $\left\{\partial_{j} \mid 1 \leqslant j \leqslant N\right\}$ in $\mathcal{L}(E)$ :

$$
\mathcal{K}(\partial):=\left\{A \in \mathcal{L}(E) \mid A \partial_{j}=\partial_{j} A \text { in } E, 1 \leqslant j \leqslant N\right\} .
$$

Let $E^{\prime}$ be the topological dual space for $E$. We introduce the set of functionals in $E^{\prime}$ "preserving" in $E$ the shifts of all functions in $E$ :

$$
\mathcal{M}(\tau):=\left\{\varphi \in E^{\prime} \mid \varphi(\tau .(f)) \in E \text { for each } f \in E\right\} .
$$

Theorem 1. Let conditions (V1) hold. The following statements are equivalent:

(i) $A \in \mathcal{K}(\tau)$.

(ii) There exists $\varphi \in \mathcal{M}(\tau)$ such that $A(f)(z)=\varphi\left(\tau_{z}(f)\right), f \in E, z \in \mathbb{C}^{N}$.

Proof. $(i) \Rightarrow(i i)$ : Let $A \in \mathcal{K}(\tau)$. Then

$$
A \tau_{z}(f)(t)=\tau_{z} A(f)(t)=\tau_{t} A(f)(z)
$$

for each $f \in E, t, z \in \mathbb{C}^{N}$. Letting $t=0$ in the latter identity, we obtain that for a continuous linear on $E$ functional $\varphi:=\delta_{0} A$, for each $f \in E, z \in \mathbb{C}^{N}$, the identity $A(f)(z)=\varphi\left(\tau_{z}(f)\right)$ holds. At that, $\varphi \in \mathcal{M}(\tau)$. Such $\varphi$ is unique since $\varphi=\delta_{0} A$. 
$($ ii $) \Rightarrow(i)$ : The continuity of the linear operator $A: E \rightarrow E$ follows the closed graph theorem [14, Ch. 6, Thm. 6.7.1]. The commuting of the operator $A$ with all translations is obvious.

We let

$$
\mathbb{N}_{0}:=\mathbb{N} \cup\{0\} ; \quad \partial^{\alpha}(f):=\frac{\partial^{|\alpha|} f}{\partial z_{1}^{\alpha_{1}} \cdots \partial z_{N}^{\alpha_{N}}}, \quad \alpha=\left(\alpha_{j}\right)_{j=1}^{N} \in \mathbb{N}_{0}^{N} ; \quad|\alpha|:=\sum_{j=1}^{N} \alpha_{j} .
$$

We introduce the functionals

$$
\varphi_{\alpha}(f):=\frac{\partial^{|\alpha|} f}{\partial z_{1}^{\alpha_{1}} \cdots \partial z_{N}^{\alpha_{N}}}(0), \quad f \in E, \quad \alpha \in \mathbb{N}_{0}^{N} .
$$

It is clear that $\varphi_{\alpha} \in E^{\prime}$ for each $\alpha \in \mathbb{N}_{0}^{N}$.

By $\tau\left(E^{\prime}, E\right)$ we denote the Mackey topology in $E^{\prime}$ defined by the natural duality between $E$ and $E^{\prime}$, see [14, Ch. 8, Sect. 8.3.3]), $E_{\tau}^{\prime}:=\left(E, \tau\left(E^{\prime}, E\right)\right)$.

Remark 3. The system $\left\{\varphi_{\alpha}: \alpha \in \mathbb{N}_{0}^{N}\right\}$ is complete in $E^{\prime}$ with each topology $\xi$ consistent with the duality between $E^{\prime}$ and $E$, that is, the closure of the linear span of this system in $\left(E^{\prime}, \xi\right)$ coincides with $E^{\prime}$. In particular, it is complete in $E_{\tau}^{\prime}$. Indeed, if $f \in E$ and $\varphi_{\alpha}(f)=0$ for each $\alpha \in \mathbb{N}_{0}^{N}$, then $f=0$.

Lemma 1. Let Condition (V1) hold. The following is true: for each $n$ there exists $l$ such that for each $k$ the identity

$$
\lim _{\varepsilon \rightarrow 0} \sup _{t \in \mathbb{C}^{N}} \frac{\sup _{|z-t| \leqslant \varepsilon}|f(z)-f(t)|}{\exp \left(v_{l, k}(t)\right)}=0
$$

holds for each function $f \in E_{n}$.

Proof. For each entire function $f$, each $z, t \in \mathbb{C}^{N}$ we have

$$
f(z)-f(t)=\sum_{j=1}^{N}\left(z_{j}-t_{j}\right) \int_{0}^{1} \partial_{j} f(t+\xi(z-t)) d \xi .
$$

We fix $n \in \mathbb{N}$. Then in view of Remark 2 (i) (see also Remark 1) we obtain that for each $k \in \mathbb{N}$ there exists a constant $C_{1}<+\infty$ such that as $\varepsilon \in(0,1]$, for each function $f \in E_{n}$ we have:

$$
\begin{aligned}
\sup _{t \in \mathbb{C}^{N}} & \left(\left(\sup _{|z-t| \leqslant \varepsilon}|f(z)-f(t)|\right) \exp \left(-v_{m(m(n)), k}(t)\right)\right) \\
\leqslant & \varepsilon \sqrt{N} \sup _{1 \leqslant j \leqslant N} \sup _{t \in \mathbb{C}^{N}}\left(\left(\sup _{|z-t| \leqslant \varepsilon}\left|\partial_{j} f(z)\right|\right) \exp \left(-v_{m(m(n)), k}(t)\right)\right) \\
\leqslant & \varepsilon \sqrt{N} \sup _{1 \leqslant j \leqslant N} \sup _{t \in \mathbb{C}^{N}}\left(\left(\left\|\partial_{j} f\right\|_{m(n), s(m(n), k)} \sup _{|w-t| \leqslant 1} \exp \left(v_{m(n), s(m(n), k)}(w)\right)\right)\right. \\
\leqslant & \left.\exp \left(-v_{m(m(n)), k}(t)\right)\right) \leqslant C_{1} \varepsilon \sqrt{N}\|f\|_{n, s_{1}},
\end{aligned}
$$

where $s_{1}:=s(n, s(m(n), k))$. Therefore, the lemma is true for $l:=m(m(n))$.

We introduce the basis in $\mathbb{C}^{N}: e_{j}=\left(\delta_{j k}\right)_{k=1}^{N}, 1 \leqslant j \leqslant N$.

Lemma 2. Let condition (V1) holds. For each $f \in E, z \in \mathbb{C}^{N}$ we have

$$
\lim _{\eta \rightarrow 0} \frac{\tau_{\eta e_{j}}(f)-f}{\eta}=\partial_{j}(f), \quad 1 \leqslant j \leqslant N,
$$

in $E$. 
This lemma is implied by Lemma 1 applied to the function $\partial_{j} f$ as $f$ and due to the identity

$$
f\left(t+\eta e_{j}\right)-f(t)=\eta \int_{0}^{1} \partial_{j} f\left(t+\xi \eta e_{j}\right) d \xi
$$

It is obvious that the functionals (delta functions) $\delta_{z}(f):=f(z), z \in \mathbb{C}^{N}$, are linear and continuous on $E$.

For $p \in \mathbb{N}$, the set $W \subset E_{p}$ by $\overline{\operatorname{aconv}(W)}^{E_{p}}$ we denote the closed absolutely convex hull of $W$ in $E_{p}$.

Lemma 3. Let conditions (V1) and (V2) hold. For each function $f \in E$ there exist $m \in \mathbb{N}$ and $p \in \mathbb{N}$ such that for each $k \in \mathbb{N}$, for

$$
V_{m, k}(f):=\left\{\exp \left(-v_{m, k}(t)\right) f(\cdot+t) \mid t \in \mathbb{C}^{N}\right\}
$$

the set $\overline{\operatorname{aconv}\left(V_{m, k}(f)\right)} E_{p}$ is compact in $E_{p}$.

Proof. Condition (V2) implies that there exists $m \in \mathbb{N}$ such that the set $V_{m, k}(f)$ is bounded in $E_{m}$ for each $k \in \mathbb{N}$. We fix $k \in \mathbb{N}$. By Remark 2 , there exists $p \in \mathbb{N}$, for which $\overline{\operatorname{aconv}\left(V_{m, k}(f)\right)} E_{p}$ is compact $E_{p}$.

For $n, k \in \mathbb{N}$ we define dual "norms" for $\|\cdot\|_{n, k}$ : for a we let $\varphi \in E_{n}^{\prime}$

$$
\|\varphi\|_{n, k}^{\prime}:=\sup _{f \in E_{n},\|f\|_{n . k} \leqslant 1}|\varphi(f)| .
$$

Theorem 2. Let conditions (V1) and (V2) hold. The following statements are equivalent:

(i) $A \in \mathcal{K}(\partial)$.

(ii) $A \in \mathcal{K}(\tau)$.

(iii) There exists $\varphi \in E^{\prime}$ such that

$$
A(f)(z)=\varphi\left(\tau_{z}(f)\right), \quad f \in E, \quad z \in \mathbb{C}^{N}
$$

Proof. $(i) \Rightarrow(i i)$ : We fix $z \in \mathbb{C}^{N}$ and $f \in E$. The identity

$$
\tau_{z}(f)(t)=\delta_{z}\left(\tau_{t}(f)\right), \quad t \in \mathbb{C}^{N}
$$

holds true. Due to Remark 3 there exists a net $\Phi_{\mu}=\sum_{\gamma \in \omega_{\mu}} b_{\gamma, \mu} \varphi_{\gamma}, \mu \in \triangle$, converging to $\delta_{z}$ in $E_{\tau}^{\prime} ; \omega_{\mu}$ are finite subsets of $\mathbb{N}_{0}^{N}$.

Let us prove that

$$
\lim _{\mu \in \triangle} \sum_{\gamma \in \omega_{\mu}} b_{\gamma, \mu} \partial^{\gamma}(f)=\tau_{z}(f)
$$

in $E$.

By Lemma 3 there exist $m, p \in \mathbb{N}$, for which $\overline{\operatorname{aconv}\left(V_{m, k}(f)\right)} E_{p}$ is compact in $E_{p}$ for each $k \in \mathbb{N}$, and therefore in $E$. Then ${\overline{\operatorname{aconv}\left(V_{m, k}(f)\right.}}^{E_{p}}$ is also $\sigma\left(E, E^{\prime}\right)$-compact in $E$. Since 
$\lim _{\mu \in \Delta} \Phi_{\mu}=\delta_{z}$ in $E_{\tau}^{\prime}$, by taking into consideration identity (2), we obtain:

$$
\begin{aligned}
A_{\mu} & :=\sup _{t \in \mathbb{C}^{N}}\left|\left(\Phi_{\mu}-\delta_{z}\right)\left(\exp \left(-v_{m, k}(t)\right) f(\cdot+t)\right)\right| \\
& =\sup _{t \in \mathbb{C}^{N}}\left(\exp \left(-v_{m, k}(t)\right)\left|\sum_{\gamma \in \omega_{\mu}} b_{\gamma, \mu} \varphi_{\gamma}(f(\cdot+t))-\tau_{z}(f)(t)\right|\right) \\
& =\sup _{t \in \mathbb{C}^{N}}\left\|\sum_{\gamma \in \omega_{\mu}} b_{\gamma, \mu} \partial^{\gamma}(f)-\tau_{z}(f)\right\|_{m, k}, \quad \mu \in \triangle
\end{aligned}
$$

Since $A_{\mu} \rightarrow 0, \mu \in \triangle$, for each $k \in \mathbb{N}$, then in $E_{m}$ and hence in $E$ we have

$$
\lim _{\mu \in \triangle} \sum_{\gamma \in \omega_{\mu}} b_{\gamma, \mu} \partial^{\gamma}(f)=\tau_{z}(f)
$$

$(i i) \Rightarrow(i)$ : This follows Lemma 2 .

The implication $(i i) \Rightarrow($ iii $)$ is true by Theorem 1 .

$($ iii $) \Rightarrow($ ii $)$ : Thanks to Theorem 1 , it is sufficient to show that $\mathcal{M}(\tau)=E^{\prime}$. We fix $\varphi \in E^{\prime}$. By Lemma 2 and Hartogs' theorem it follows that $\varphi\left(\tau_{z}(f)\right)$ is an entire function in $z \in \mathbb{C}^{N}$. We take $n \in \mathbb{N}$ and choose $m$ by $n$ via condition (V2). Since $\varphi \in E^{\prime}$, for some $k$, the functional $\varphi$ is continuous in the sense of the norm $\|\cdot\|_{m, k}$. We introduce $s$ and $C$ by Condition (V2). Then for $f \in E_{n}$

$$
\begin{aligned}
\sup _{z \in \mathbb{C}^{N}} \frac{\left|\varphi\left(\tau_{z}(f)\right)\right|}{\exp \left(v_{m, k}(z)\right)} & \leqslant\|\varphi\|_{m, k}^{\prime} \sup _{z \in \mathbb{C}^{N}} \frac{\|f(\cdot+z)\|_{m, k}}{\exp \left(v_{m, k}(z)\right)} \\
& \leqslant\|\varphi\|_{m, k}^{\prime} \sup _{z \in \mathbb{C}^{N}} \sup _{t \in \mathbb{C}^{N}} \frac{|f(t+z)|}{\exp \left(v_{m, k}(t)+v_{m, k}(z)\right)} \leqslant e^{C}\|\varphi\|_{m, k}^{\prime} \sup _{t, z \in \mathbb{C}^{N}} \frac{|f(t+z)|}{\exp \left(v_{n, s}(z+t)\right)} \\
& =e^{C}\|\varphi\|_{m, k}^{\prime}\|f\|_{n, s}<+\infty .
\end{aligned}
$$

Therefore, $\varphi(\tau .(f)) \in E$. Moreover, the latter inequality implies the continuity of the operator $A$ from $E_{n}$ into $E_{m}$, and hence, from $E$ into $E$. The continuity of the linear operator $A: E \rightarrow E$ is also implied by the closed graph theorem [14, Ch. 6, Thm. 6.7.1].

\subsection{Example. Immediate continuity of linear operators commuting with differ-} entiation operators. We provide the example of space $E$, for which the sets $\mathcal{K}(\partial)$ and $\mathcal{K}(\tau)$ coincide but Statements (i) and (ii) of Theorem 2 are not equivalent to (iii), that is, $M(\tau)$ is an eigenspace in $E^{\prime}$. At that we prove a result on immediate continuity of linear operators commuting with the operators $\partial_{j}, 1 \leqslant j \leqslant N$.

Let $\mathcal{D}:=\mathcal{D}\left(\mathbb{R}^{N}\right)$ be the space of all infinitely differentiable compactly supported in $\mathbb{R}^{N}$ functions equipped by the natural topology of (LF)-space. We let

$$
\langle t, z\rangle=\sum_{j=1}^{N} t_{j} z_{j}, \quad t=\left(t_{j}\right)_{j=1}^{N}, \quad z=\left(z_{j}\right)_{j=1}^{N} \in \mathbb{C}^{N} .
$$

As it is known, the Fourier-Laplace transform

$$
\mathcal{F}(h)(z):=\int_{\mathbb{R}^{N}} h(x) e^{-i\langle x, z\rangle} d x, \quad z \in \mathbb{C}^{N},
$$

is the topological isomorphism of $\mathcal{D}$ on the (LF)-space $E_{\mathcal{D}}:=E$ defined, as above, by the functions $v_{n, k}(z):=\exp (n|\operatorname{Im} z|-k \log (1+|z|)), z \in \mathbb{C}^{N}, n, k \in \mathbb{N}$. This sequence satisfies Condition (V1) but does not Condition (V2). 
We let $e_{z}(t):=e^{-i\langle z, t\rangle}, t, z \in \mathbb{C}^{N}$. We introduce the operators of multiplication by independent variables $x_{j}: M_{j}(f)(x):=x_{j} f(x), x \in \mathbb{R}^{N}$. By $I$ we denote the identity mapping in $\mathcal{D}$.

Proposition 1. For each linear operator $A: \mathcal{D} \rightarrow \mathcal{D}$ the following statements are equivalent:

(i) A commutes with each operator $M_{j}, 1 \leqslant j \leqslant N$.

(ii) There exists a function $a \in C^{\infty}\left(\mathbb{R}^{N}\right)$ such that $A(f)=a f, f \in \mathcal{D}$.

Proof. The implication $(i i) \Rightarrow(i)$ is obvious.

$(i) \Rightarrow(i i)$ : Let a linear operator $A: \mathcal{D} \rightarrow \mathcal{D}$ be such that $A M_{j}=M_{j} A, 1 \leqslant j \leqslant N$, on $\mathcal{D}$.

We choose $f \in \mathcal{D}$ and a function $h \in D$ such that $h \equiv 1$ on $\operatorname{supp} f$. Then $f=f h$. For $x=\left(x_{j}\right)_{j=1}^{N}, \lambda=\left(\lambda_{j}\right)_{j=1}^{N} \in \mathbb{R}^{N}$ we let

$$
\begin{aligned}
& f_{j, \lambda}(x):=f\left(\lambda_{1}, \ldots, \lambda_{j-1}, x_{j}, \ldots, x_{N}\right)-f\left(\lambda_{1}, \ldots, \lambda_{j-1}, \lambda_{j}, x_{j+1}, \ldots, x_{N}\right), \quad 2 \leqslant j \leqslant N ; \\
& f_{1, \lambda}(x):=f(x)-f\left(\lambda_{1}, x_{2}, \ldots, x_{N}\right) .
\end{aligned}
$$

It is clear that

$$
f=f(\lambda) h+\sum_{j=1}^{N} f_{j, \lambda} h
$$

By the Taylor formula we have

$$
\begin{aligned}
f_{j, \lambda}(x)= & \left(x_{j}-\lambda_{j}\right) \partial_{j} f\left(\lambda_{1}, \ldots, \lambda_{j}, x_{j+1}, \ldots, x_{N}\right) \\
& +\left(x_{j}-\lambda_{j}\right)^{2} \int_{0}^{1}(1-s) \partial_{j}^{2} f\left(\lambda_{1}, \ldots,(1-s) \lambda_{j}+s x_{j}, x_{j+1}, \ldots, x_{N}\right) d s \\
= & \left(x_{j}-\lambda_{j}\right) \widetilde{f}_{j, \lambda}(x), \quad \lambda \in \mathbb{R}^{N}, \quad 1 \leqslant j \leqslant N,
\end{aligned}
$$

where the function

$$
\begin{aligned}
\widetilde{f}_{j, \lambda}(x):= & \partial_{j} f\left(\lambda_{1}, \ldots, \lambda_{j}, x_{j+1}, \ldots, x_{N}\right) \\
& +\left(x_{j}-\lambda_{j}\right) \int_{0}^{1}(1-s) \partial_{j}^{2} f\left(\lambda_{1}, \ldots,(1-s) \lambda_{j}+s x_{j}, x_{j+1}, \ldots, x_{N}\right) d s
\end{aligned}
$$

is infinitely differentiable in $\mathbb{R}^{N}$.

Let $\delta_{\lambda}(f)=0$, that is, $f(\lambda)=0$. Since $f_{j, \lambda} h=\left(M_{j}-\lambda_{j} I\right)\left(\widetilde{f}_{j, \lambda} h\right), 1 \leqslant j \leqslant N$, by (3) we get:

$$
A(f)(\lambda)=\sum_{j=1}^{N} A\left(M_{j}-\lambda_{j} I\right)\left(\tilde{f}_{j, \lambda} h\right)(\lambda)=\sum_{j=1}^{N}\left(\lambda_{j} A\left(\widetilde{f}_{j, \lambda} h\right)(\lambda)-\lambda_{j} A\left(\widetilde{f}_{j, \lambda} h\right)(\lambda)\right)=0 .
$$

On $\mathcal{D}$ we define the linear functional $\gamma_{\lambda}(f):=A(f)(\lambda), f \in \mathcal{D}$. Then, as it has been shown above, $\operatorname{Ker} \delta_{\lambda} \subset \operatorname{Ker} \gamma_{\lambda}$. This is why for each $\lambda \in \mathbb{R}^{N}$ there exists $a(\lambda) \in \mathbb{C}$ such that $A(f)(\lambda)=$ $a(\lambda) f(\lambda)$ for each function $f \in \mathcal{D}$. It is obvious that $a \in C^{\infty}\left(\mathbb{R}^{N}\right)$.

Corollary 1. (i) Each linear operator $A: \mathcal{D} \rightarrow \mathcal{D}$ commuting with each operator $M_{j}, 1 \leqslant$ $j \leqslant N$, is continuous.

(ii) Each linear operator $A: E_{\mathcal{D}} \rightarrow E_{\mathcal{D}}$ commuting with each operator $\partial_{j}, 1 \leqslant j \leqslant N$, is continuous.

We observe that in [9, Lm. 1.7], [4] there were described linear operators commuting with the multiplication by an independent variable in the spaces of holomorphic functions of one variable and the immediate continuity of these operators was proved.

It follows from Proposition 1 and standard properties of Fourier-Laplace transform that the belonging of the operator $A$ to the set $\mathcal{K}(\partial)$ is the equivalent to the existence of a (unique) 
function $a \in C^{\infty}\left(\mathbb{R}^{N}\right)$ such that $A=\mathcal{F} M_{a} \mathcal{F}^{-1}$, where $M_{a}$ is the operator of the multiplication by the function $a$. For $a \in C^{\infty}\left(\mathbb{R}^{N}\right), f \in E_{\mathcal{D}}, z \in \mathbb{C}^{N}$, we have

$$
\mathcal{F} M_{a} \mathcal{F}^{-1}(f)(z)=\int_{\mathbb{R}^{N}} e^{-i\langle x, z\rangle} a(x) \mathcal{F}^{-1}(f)(x) d x=a\left(\mathcal{F}^{-1}(f) e_{z}\right) .
$$

At that, $a$ is identified with the regular generalized function defined by $a$. On the other hand, if $\mathcal{F}^{\prime}: E_{\mathcal{D}}^{\prime} \rightarrow \mathcal{D}^{\prime}$ is the mapping adjoint for $\mathcal{F}: \mathcal{D} \rightarrow E_{\mathcal{D}}$, then

$$
\left(\left(\mathcal{F}^{\prime}\right)^{-1}(a)\right)_{t}(f(t+z))=a\left(\mathcal{F}^{-1}(f) e_{z}\right) .
$$

This implies that in this case $M(\tau)$ is "much narrower" than $E^{\prime}$, namely,

$$
M(\tau)=\left\{\varphi \in E^{\prime} \mid \mathcal{F}^{\prime}(\varphi) \in C^{\infty}\left(\mathbb{R}^{N}\right)\right\} .
$$

2. Multiplication $\odot$ in $E^{\prime}$ And the properties of algebra $\left(E^{\prime}, \odot\right)$

Hereafter we assume that the sequence $\left(v_{n, k}\right)_{n, k \in \mathbb{N}}$ satisfies Conditions (V1) and (V2). Let us introduce a multiplication $\odot$ in $E^{\prime}$. For $\varphi, \psi \in E^{\prime}$ we let

$$
(\varphi \odot \psi)(f):=\varphi_{t}\left(\psi\left(\tau_{t}(f)\right)\right), \quad f \in E .
$$

Thanks to Theorem 2 and the identity $\varphi=\delta_{0} \omega(\varphi)$, the operation $\odot$ is well-defined and the mapping $\omega: E^{\prime} \rightarrow \mathcal{K}(\partial)$,

$$
\omega(\varphi)(f)(z):=\varphi\left(\tau_{z}(f)\right), \quad \varphi \in E^{\prime}, \quad f \in E, \quad z \in \mathbb{C}^{N},
$$

is bijective. Since for each $\varphi, \psi \in E^{\prime}, f \in E, z \in \mathbb{C}^{N}$,

$$
\begin{aligned}
\omega(\varphi \odot \psi)(f)(z) & =(\varphi \odot \psi)\left(\tau_{z}(f)\right)=\varphi_{t}\left(\psi\left(\tau_{t}\left(\tau_{z}(f)\right)\right)\right)=\varphi\left(\omega(\psi)\left(\tau_{z}(f)\right)\right) \\
& =\varphi\left(\tau_{z}(\omega(\psi))(f)\right)=\omega(\varphi)(\omega(\psi)(f))(z)=\omega(\varphi) \omega(\psi)(f)(z),
\end{aligned}
$$

then $\omega$ is the isomorphism of algebras $\left(E^{\prime}, \odot\right)$ and $\mathcal{K}(\partial)$; in the latter the multiplication is the composition of the operators. This implies that the multiplication $\odot$ in $E^{\prime}$ is associative.

The following Lemma 4 implies that the composition of the operators in $\mathcal{K}(\partial)$ is commutative.

Lemma 4. Let Conditions (V1) and (V2) hold.

(i) If the net $\Psi_{\nu} \in E^{\prime}, \nu \in \Delta$, converges to $\psi \in E^{\prime}$ in $E_{\tau}^{\prime}$, then for each function $f \in E$ in $E_{\tau}^{\prime}$ there exists the limit $\lim _{\nu \in \Delta}\left(\Psi_{\nu}\right)_{z}(f(\cdot+z))$ and it is equal to $\psi_{z}(f(\cdot+z))$.

(ii) The algebra $\left(E^{\prime}, \odot\right)$ is commutative.

Proof. (i): We fix $f \in E$. By Lemma 3 there exist $m \in \mathbb{N}$ and $p \in \mathbb{N}$, for which the set $\mathcal{V}:=\overline{\operatorname{aconv}\left(V_{m, k}(f)\right)} E_{p}$ is compact in $E_{p}$, where

$$
V_{m, k}(f):=\left\{\exp \left(-v_{m, k}(t)\right) \tau_{t}(f) \mid t \in \mathbb{C}^{N}\right\} .
$$

Since $E_{p}$ is compactly embedded into $\left(E, \sigma\left(E, E^{\prime}\right)\right)$, then $\mathcal{V}$ is also $\sigma\left(E, E^{\prime}\right)$-compact. Therefore, the net $\left\{\Psi_{\nu} \mid \nu \in \Delta\right\}$ converges to $\psi$ uniformly on $\mathcal{V} \supseteq V_{m, k}(f)$. This is why

$$
\sup _{t \in \mathbb{C}^{N}}\left|\exp \left(-v_{m, k}(t)\right)\left(\Psi_{\nu}-\psi\right)_{z}(f(t+z))\right| \underset{\nu \in \Delta}{\longrightarrow} 0
$$

and

$$
p_{m, k}\left(\left(\Psi_{\nu}-\psi\right)_{z}(f(\cdot+z))\right) \mid \underset{\nu \in \Delta}{\longrightarrow} 0 .
$$

Thus, in $E_{m}$, and hence in $E$ the identity

$$
\lim _{\nu \in \Delta}\left(\Psi_{\nu}\right)_{z}(f(\cdot+z))=\psi_{z}(f(\cdot+z))
$$

holds. 
(ii): We take $\varphi, \psi \in E^{\prime}$. Since by Remark 3 the set of the functionals $\left\{\varphi_{\alpha} \mid \alpha \in \mathbb{N}_{0}^{N}\right\}$ is complete in $E_{\tau}^{\prime}$, there exists nets $\Phi_{\mu} \in \operatorname{span}\left\{\varphi_{\alpha} \mid \alpha \in \mathbb{N}_{0}^{N}\right\}, \mu \in \Lambda$, and $\Psi_{\nu} \in \operatorname{span}\left\{\varphi_{\alpha} \mid \alpha \in \mathbb{N}_{0}^{N}\right\}$, $\nu \in \Delta$, converging to $E_{\tau}^{\prime}$ in $\varphi$ and $\psi$, respectively. We note that for each $\alpha, \beta \in \mathbb{N}_{0}^{N}$, each function $g \in H\left(\mathbb{C}^{N}\right)$ we have

$$
\left(\varphi_{\alpha}\right)_{z}\left(\left(\varphi_{\beta}\right)_{t}(g(t+z))\right)=\left(\varphi_{\beta}\right)_{t}\left(\left(\varphi_{\alpha}\right)_{z}(g(t+z))\right) .
$$

In view of Statement (i), for each function $f \in E$ we get

$$
\begin{aligned}
(\varphi \odot \psi)(f) & =\varphi_{t}\left(\psi_{z}(f(t+z))\right)=\varphi_{t}\left(\lim _{\nu \in \Delta}\left(\Psi_{\nu}\right)_{z}(f(t+z))\right) \\
& =\lim _{\nu \in \Delta} \varphi_{t}\left(\left(\Psi_{\nu}\right)_{z}(f(t+z))\right)=\lim _{\nu \in \Delta}\left(\lim _{\mu \in \Lambda}\left(\Phi_{\mu}\right)_{t}\left(\left(\Psi_{\nu}\right)_{z}(f(t+z))\right)\right. \\
& =\lim _{\nu \in \Delta}\left(\lim _{\mu \in \Lambda}\left(\Psi_{\nu}\right)_{z}\left(\left(\Phi_{\mu}\right)_{t}(f(t+z))\right)=\lim _{\nu \in \Delta}\left(\left(\Psi_{\nu}\right)_{z}\left(\lim _{\mu \in \Lambda}\left(\Phi_{\mu}\right)_{t}(f(t+z))\right)\right.\right. \\
& =\lim _{\nu \in \Delta}\left(\Psi_{\nu}\right)_{z}\left(\varphi_{t}(f(t+z))\right)=\psi_{z}\left(\varphi_{t}(f(t+z))\right)=(\psi \odot \varphi)(f) .
\end{aligned}
$$

Remark 4. Non-zero multiplicative linear $\sigma\left(E^{\prime}, E\right)$-continuous functionals on $\left(E^{\prime}, \odot\right)$, that is, the non-zero multiplicative functionals of the form $\varphi \mapsto \varphi(g), \varphi \in E^{\prime}$, where $g$ is a fixed element of $E$, are exactly those determined by the function $g(z)=e^{\langle\nu, z\rangle}, \nu \in \mathbb{C}^{N}$ provided it belongs to $E$. This is why for each such function $g$, the hypersubspace $H_{g}:=\left\{\varphi \in E^{\prime} \mid \varphi(g)=0\right\}$ is a $\sigma\left(E^{\prime}, E\right)$-closed maximal ideal in $\left(E^{\prime}, \odot\right)$.

Proof. It is obvious that each functional $G(\varphi):=\varphi_{z}\left(e^{\langle\nu, z\rangle}\right)$ with $e^{\langle\nu, \cdot} \in E$ is multiplicative. Let $G(\varphi)=\varphi(g), \varphi \in E^{\prime}$, for some function $g \in E$. Then for each $\lambda, \mu \in \mathbb{C}^{N}$ the identity

$$
g(\lambda) g(\mu)=G\left(\delta_{\lambda}\right) G\left(\delta_{\mu}\right)=G\left(\delta_{\lambda} \odot \delta_{\mu}\right)=g(\lambda+\mu)
$$

holds. This implies that a (non-zero) function $g$ is the function $e^{\langle\nu, z\rangle}, z \in \mathbb{C}^{N}$, for some $\nu \in \mathbb{C}^{N}$. In fact, this has been proved in the proof of Lemma 9.24 in [8].

2.1. Topological isomorphism of $E^{\prime}$ and $\mathcal{K}(\partial)$. We consider the case when the considered spaces are equipped by weak and the corresponding topologies. By the symbol $\mathcal{K}_{\sigma}(\partial)$ we denote the space $\mathcal{K}(\partial)$ with the weak operator topology, that is, with the topology of pointwise convergence, when the weak topology $\sigma\left(E, E^{\prime}\right)$ is introduced in $E$ (see [13, Ch.III, Sect. 3, Ex.4 (a)]). We note that due to the barreledness of $E$, the space of all linear weakly continuous operators in $E$ coincides algebraically with $\mathcal{L}(E)$ [14, Ch. 8, Sect. 8.6, Thm. 8.6.1]. In what follows we denote $\sigma:=\sigma\left(E^{\prime}, E\right)$.

Theorem 3. The mapping $\omega$ is a topological isomorphism of $\left(E^{\prime}, \sigma\right)$ on $\mathcal{K}_{\sigma}(\partial)$.

Proof. For each finite set $M \subset E$, each $\varphi \in E^{\prime}$, the identity

$$
\sup _{f \in M}|\varphi(f)|=\sup _{f \in M}\left|\delta_{0}(\omega(\varphi)(f))\right|
$$

holds. Therefore, the mapping $\omega^{-1}: \mathcal{K}_{\sigma}(\partial) \rightarrow\left(E^{\prime}, \sigma\right)$ is continuous.

The continuity of the mapping $\omega:\left(E^{\prime}, \sigma\right) \rightarrow \mathcal{K}_{\sigma}(\partial)$ is implied by the fact that in view of Lemma 4 (ii), for each finite sets $M \subset E, P \subset E^{\prime}$, each $\varphi \in E^{\prime}$, we have

$$
\begin{aligned}
\sup _{f \in M, \psi \in P}|\psi(\omega(\varphi)(f))| & =\sup _{f \in M, \psi \in P}\left|\psi_{z}\left(\varphi_{t}(f(t+z))\right)\right|=\sup _{f \in M, \psi \in P}|(\psi \odot \varphi)(f)| \\
& =\sup _{f \in M, \psi \in P}|(\varphi \odot \psi)(f)|=\sup _{f \in M, \psi \in P}\left|\varphi_{t}\left(\psi_{z}(f(t+z))\right)\right|=\sup _{v \in \widetilde{M}}|\varphi(v)|,
\end{aligned}
$$

where $\widetilde{M}$ is a finite subset of $E$ consisting of the functions $\psi_{z}(f(t+z))$ of the variable $t, f \in M$, $\psi \in P$. 
Let $\mathcal{P}(\partial):=\operatorname{span}\left\{\partial^{\alpha} \mid \alpha \in \mathbb{N}_{0}^{N}\right\}$, that is, $\mathcal{P}(\partial)$ is the set of all polynomials of the operators $\partial_{j}, 1 \leqslant j \leqslant N$.

Corollary 2. The set $\mathcal{P}(\partial)$ is dense in $\mathcal{K}(\partial)$ equipped with the topology of pointwise convergence.

Proof. Since by Remark 3 the set $\left\{\varphi_{\alpha} \mid \alpha \in \mathbb{N}_{0}^{N}\right\}$ is dense in $\left(E^{\prime}, \sigma\right)$, by Theorem 3 the set $\left\{\partial^{\alpha}=\omega\left(\varphi_{\alpha}\right) \mid \alpha \in \mathbb{N}_{0}^{N}\right\}$ is dense in $\mathcal{K}_{\sigma}(\partial)$. This is why for each $f \in E$ in the space $E$, the set $\mathcal{P}(\partial)(f):=\{A(f) \mid A \in \mathcal{P}(\partial)\}$ is weakly dense in $\mathcal{K}(\partial)(f):=\{A(f) \mid A \in \mathcal{K}(\partial)\}$. Hence, for each $f \in E$, the set $\mathcal{P}(\partial)(f)$ is dense in $\mathcal{K}(\partial)(f)$ also in the sense of the topology in $E$. Thus, $\mathcal{P}(\partial)$ is dense in $\mathcal{K}(\partial)$ equipped with the topology of the pointwise convergence.

Theorem 3 implies the following result clarifying the nature of the spaces $E$, for which each operator in $\mathcal{K}(\partial)$ is an infinite order differential operator with constant coefficients.

Corollary 3. The following statements are equivalent:

(i) $\left\{\varphi_{\alpha} \mid \alpha \in \mathbb{N}_{0}^{N}\right\}$ is the basis in $\left(E^{\prime}, \sigma\left(E^{\prime}, E\right)\right)$.

(ii) $\left\{\partial^{\alpha} \mid \alpha \in \mathbb{N}_{0}^{N}\right\}$ is the basis in $\mathcal{K}_{\sigma}(\partial)$.

Example 1. Let $Q$ be a convex locally closed set in $\mathbb{C}^{N}$ containing zero, $\left(Q_{n}\right)_{n \in \mathbb{N}}$ be a fundamental sequence of compact subsets in $Q$; without loss of generality we assume that all compact sets $Q_{n}$ are convex and $Q_{n} \subset Q_{n+1}$ for each $n \in \mathbb{N}$. By $H_{Q_{n}}$ denote the support function of $Q_{n}$, that is, $H_{Q_{n}}(z):=\sup _{t \in Q_{n}} \operatorname{Re}\langle t, z\rangle, z \in \mathbb{C}^{N}$. The sequence

$$
v_{n, k}(z):=H_{Q_{n}}(z)+\frac{|z|}{k}, \quad z \in \mathbb{C}^{N}, \quad n, k \in \mathbb{N},
$$

satisfies Conditions (V1) and (V2). In this case, via the Laplace transform, $E$ is the strong dual space for the space $H(Q)$ of the germs of all functions holomorphic on $Q$ [17, Lm. 1.10].

We note that $Q$ can be a convex domain or a convex closed set in $\mathbb{C}^{N}$. If $Q=\mathbb{R}^{N}$, then $H(Q)=\mathcal{A}\left(\mathbb{R}^{N}\right)$ is the space of real analytic in $\mathbb{R}^{N}$ functions.

Example 2. Let

$$
v_{n, k}(z):=n(|\operatorname{Im} z|+\log (1+|z|)), \quad z \in \mathbb{C}^{N}, \quad n, k \in \mathbb{N} ;
$$

the functions $v_{n, k}$ are independent of $k$. The sequence $\left(v_{n, k}\right)_{n, k \in \mathbb{N}}$ satisfies Conditions (V1) and (V2). The space $E$ defined by this sequence provides via the Laplace-Fourier transform the strong dual space for the Fréchet space $C^{\infty}\left(\mathbb{R}^{N}\right)$ of all infinitely differentiable in $\mathbb{R}^{N}$ functions.

\section{BIBLIOGRAPHY}

1. A.V. Bratishchev, Yu.F. Korobeinik. General form of linear operators which commute with the operation of differentiation // Matem. Zamet. 12:2, 187-195 (1972). [Math. Notes. 12:2, 547-551 (1972).]

2. O.A. Ivanova, S.N. Melikhov. On operators which commute with the Pommiez type operator in weighted spaces of entire functions // Alg. Anal. 28:2, 114-137 (2016). [St.-Petersburg Math. J. 28:2, 209-224 (2017).]

3. Yu.F. Korobeinik. On one class of linear operators // Godishnik Vyssh. Tekh. Ucheb. Zav. Matem. 9:3, 23-33 (1973). (in Russian).

4. Yu.F. Korobeinik. On representation of linear operators commuting with the operator of multiplication // Anal. Math. 32:2, 123-153 (2006). (in Russian).

5. Yu.F. Korobeinik. On solvability of some classes of linear operator equations in complex domain. Southern Federal Univ. Publ., Rostov-on-Don (2009). (in Russian).

6. Yu.F. Korobeinik, V.V. Morzhakov. The general form of isomorphisms commuting with differentiation in spaces of entire functions of slow growth // Matem. Sborn. 91(133):4, 475-487 (1973). [Math. USSR-Sb. 20:4, 493-505 (1973).] 
7. B.Ya. Levin. Distribution of zeros of entire functions. GITTL, Moscow (1956). [Amer. Math. Soc., Providence, R.I. (1964).]

8. P. Lelong, L. Gruman. Entire functions of several complex variables. Grundlehren der Mathematischen Wissenschaften. 282. Springer, Berlin (1986).

9. S.N. Melikhov. On the left inverse to the restriction operator on weighted spaces of entire functions // Alg. Anal. 14:1, 99-113 (2002). [St.-Petersburg Math. J. 14:1, 75-99 (2003).]

10. V.V. Napalkov. Operators, commuting with differentiation, in spaces of functions of several variables // Matem. Zamet. 24:6, 829-838 (1978). [Math. Notes. 24:6, 939-949 (1978).]

11. V.M. Trutnev. Inhomogeneous convolution equations in some spaces of entire functions of exponential type // in "Complex analysis and differential equations". Krasnoyarsk State Univ., Krasnoyarsk (1996). (in Russian).

12. V.M. Trutnev. Convolution equations in spaces of entire functions of exponential type // Itogi Nauki Tekhn. Sovrem. Matem. Pril. Temat. Obzory. 108, 158-180 (2006). [J. Math. Sci. 120:6, 1901-1915 (2004).]

13. H.H. Schaefer. Topological vector spaces. The Macmillian Company, New York (1966).

14. P. Edwards. Functional analysis. Theory and applications. Holt Rinehart and Winston, New York (1965).

15. G. Godefroy, J.H. Shapiro. Operators with dense, invariant, cyclic vector manifolds // J. Funct. Anal. 98:2, 229-269 (1991).

16. A. Martineau. Équations différentielles d'ordre infini // Bull. Soc. Math. France. 95, 109-154 (1967).

17. S.N. Melikhov, S. Momm. Analytic solutions of convolution equations on convex sets with an obstacle in the boundary // Math. Scand. 86:2, 293-319 (2000).

Ol'ga Alexandrovna Ivanova,

Southern Federal University,

Institute of Mathematics,

Mechanics and Computer Sciences

named after I.I. Vorovich,

Mil'chakova str. 8a,

344090, Rostov-on-Don, Russia

E-mail: ivolga@sfedu.ru

Sergej Nikolaevich Melikhov,

Southern Federal University,

Institute of Mathematics,

Mechanics and Computer Sciences

named after I.I. Vorovich,

Mil'chakova str. 8a,

344090, Rostov-on-Don, Russia

South Mathematical Institute,

Vladikavkaz Scientific Center, RAS,

Markus str. 22,

362027, Vladikavkaz, Russia

E-mail: melih@math.rsu.ru

Yuri Nikolaevich Melikhov,

Military Academy of ASD

named after G.K. Zhukov,

Zhigareva str. 50,

170022, Tver, Russia

E-mail: melikhow@mail.ru 